\title{
Physical parameters and emission mechanism in gamma-ray bursts
}

\author{
E. V. Derishev ${ }^{1}$, V. V. Kocharovsky ${ }^{1,2}$, and Vl. V. Kocharovsky ${ }^{1}$ \\ 1 Institute of Applied Physics, Russian Academy of Science, 46 Ulyanov st., 603950 Nizhny Novgorod, Russia \\ 2 Dept. of Physics, Texas A\&M University, College Station, TX 77843-4242, USA \\ e-mail: kochar@appl.sci-nnov.ru
}

Received 13 June 2000 / Accepted 7 March 2001

\begin{abstract}
Detailed information on the physical parameters in the sources of cosmological Gamma-Ray Bursts (GRBs) is obtained from few plausible assumptions consistent with observations. We consider monoenergetic injection of electrons and let them cool self-consistently, taking into account Klein-Nishina cut-off in electronphoton scattering. The general requirements posed by the assumptions on the emission mechanism in GRBs are formulated. It is found that the observed radiation in the sub-MeV energy range is generated by the synchrotron emission mechanism, though about ten per cent of the total GRB energy should be converted via the inverse Compton (IC) process into the ultra-hard spectral domain (above $100 \mathrm{GeV}$ ). We estimate the magnetic field strength in the emitting region, the Lorentz factor of accelerated electrons, and the typical energy of IC photons. We show that there is a synchrotron-self-Compton constraint which limits the parameter space available for GRBs that are radiatively efficient in the sub- $\mathrm{MeV}$ domain. This concept is analogous to the line-of-death relation existing for pulsars and allows us to derive the lower limits on both GRB duration and the timescale of GRB variability. The upper limit on the Lorentz factor of GRB fireballs is also found. We demonstrate that steady-state electron distribution consistent with the Compton losses may produce different spectral indices, e.g., 3/4 as opposed to the figure $1 / 2$ widely discussed in the literature. It is suggested that the changes in the decline rate observed in the lightcurves of several GRB afterglows may be due to either a transition to efficient IC cooling or the time evolution of Klein-Nishina and/or Compton spectral breaks, which are the general features of self-consistent electron distribution.
\end{abstract}

Key words. radiation mechanisms: non-thermal - ISM: jets and outflows - gamma rays: bursts - gamma rays: theory

\section{Introduction}

At present, there is a tendency to divide the problem of Gamma-Ray Bursts into two separate problems: the nature of the central engine and the origin of GRB emission. While the former question is still far from being settled, the multi-wavelength observations (e.g., Frail et al. 1997; Metzger et al. 1997; Reichart 1997; Waxman 1997; see also Piran 1999 for a review), which become available in the past few years, give strong support to the fireball shock model (Rees \& Mészáros 1992, 1994). In its most general form, this model considers GRB emission as the result of energy dissipation in relativistic shock waves and assumes that all the energy is initially accumulated in the form of ultrarelativistic outflow (fireball) with a Lorentz factor $\Gamma$ greater than 100 (Baring \& Harding 1995). The fraction

Send offprint requests to: E. V. Derishev, e-mail: derishev@appl.sci-nnov.ru of energy carried directly by the emission from the fireball photosphere is small unless $\Gamma \gtrsim 10^{5}$ (Derishev et al. 1999).

The fireball model became widespread and almost undisputed, but in its details there is no agreement among theorists. Even the principal question about the main emission mechanism has not yet received a definite answer, although synchrotron and inverse Compton emission are the favourites (e.g., Paczyński \& Rhoads 1993; Mészáros et al. 1993; Sari et al. 1998). The existing uncertainty complicates the correct interpretation of the growing number of dissimilar multi-wavelength observations.

In this paper we derive constraints on the physical parameters in the GRB emitting region and present new arguments in favour of the synchrotron emission mechanism. There are two arguments widely discussed in the literature in this connection. First, the observed polarization in afterglow emission (Covino et al. 1999; Wijers et al. 1999 ) is thought to be indicative of synchrotron mechanism. Second, it was argued that the electron cooling 
time is too long to be compatible with high radiative efficiency if one assumes self-Compton mechanism as the main source of sub-Mev radiation (Piran 1999). Both arguments are not very restrictive because: a) polarization may be produced by inverse Compton scattering as well if the radiation background and/or electron distribution is anisotropic, b) the strongest limit on the bulk Lorentz factor imposed by cooling time considerations is for the external shock model, $\Gamma<300-400$, which is still above the generally accepted lower limit of $\sim 100$. In this paper we show that the additional requirement of high efficiency in sub-MeV gamma-rays relative to other spectral domains pushes the upper limit on $\Gamma$ to an unacceptable value of $\sim 25$ unless the sub-MeV radiation is generated by synchrotron rather than by a self-Compton mechanism.

There is also an argument against a synchrotron-selfCompton model: it is sometimes considered as insufficiently flexible to account for different low-energy spectral indices provided the electrons form a cooling distribution. Below we show that this argument is rather weak since a broad range of spectral indices may be accommodated within the framework of synchrotron-self-Compton model as long as the cooling distribution is calculated consistently with total losses.

We introduce the minimum number of a priori assumptions and avoid the considerations which would require the electron acceleration mechanism to be specified. All the assumptions used are explicitly listed in the second section, followed by a brief discussion and a comparison with other approaches frequently used in the literature. The basic analysis is presented in the third section, and the implications are discussed in the forth section.

For numerical estimates we take GRB energy $E_{\mathrm{GRB}}=$ $10^{52} \mathrm{erg}$ and duration $t_{\mathrm{GRB}}=10 \mathrm{~s}$. All other values are measured in the shock comoving frame unless the opposite is stated. It should be noted that for two bursts, GRB 971214 and GRB 990123, the estimated value of energy release approaches $10^{54}$ ergs assuming isotropic emission. Since it is very divergent from the average value $\left(10^{51}-10^{52}\right.$ ergs) inferred from the analysis of the $\log N$ $\log S$ curve (Reichart \& Mészáros 1997), these bursts may be of different origin.

\section{Model assumptions}

1. Most GRB emission is in the form of soft gamma-rays, i.e., below several $\mathrm{MeV}$. - It would naturally explain why GRB phenomenon was first detected in this spectral range. The observation of optical emission from GRB 990123 (Akerlof et al. 1999), made simultaneously with the burst of gamma-rays, confirms our first assumption. However, observations do not exclude the possibility that the main part of GRB emission may be concentrated in photons with energies greater than $1 \mathrm{TeV}$, and hence escapes detection because photons in this energy range are strongly absorbed by infrared background radiation (Primack et al. 1999).
2. A major part of the fireball kinetic energy is converted into electromagnetic radiation during the main GRB pulse. - GRB afterglows decline faster than $t^{-1}$ and do not contribute significantly to the total energy budget (Galama et al. 1998). However, it is possible that the adiabatic regime of the shock deceleration persists for a very long time, so that the bulk of GRB energy has never been observed. Another reason to make the second assumption is not to increase the energy requirements, which are already rather restrictive unless the fireball forms a narrow jet (e.g., Mészáros et al. 1999).

3. GRB emission is generated by relativistic electrons and positrons, which are continuously injected with the initial Lorentz factor $\gamma_{\mathrm{p}}$. Acceleration of an electron takes a very short time compared to the shock expansion timescale and electron cooling timescale. - This same assumption implies that we refer to the synchrotron-self-Compton model of GRB emission. Comparison with the other emission models favours the synchrotron-self-Compton one, which indeed can account for high radiative efficiency.

If one does not postulate the high efficiency of energy conversion into electromagnetic radiation, then the number of available emission mechanisms increases dramatically (e.g., Shaviv \& Dar 1995; Panaitescu et al. 1999) and it becomes almost impossible to choose only one of them on the basis of limited observational data. At the same time, any model with low efficiency is problematic, as it can hardly compete with blackbody photospheric emission in the $10-100 \mathrm{keV}$ range.

Evolution of the Lorentz factor $\gamma_{\mathrm{e}}$ (index e denotes both electrons and positrons) is governed by the acceleration and losses. The Lorentz factor grows until the losses compensate the acceleration term. Thus, electron acceleration is terminated at approximately the same Lorentz factor, $\gamma_{p}$, which is close to the value that equilibrates acceleration and radiative losses.

Some authors, however, prefer to consider electron injection with a power-law spectrum, $\mathrm{d} n_{\mathrm{e}}\left(\gamma_{\mathrm{e}}\right) / \mathrm{d} t \propto \gamma_{\mathrm{e}}^{-q}$, extending from $\gamma_{\min }$ to infinity (e.g., Sari et al. 1998). The spectrum is integrable $(q>2)$ and hence most of the energy is concentrated near $\gamma_{\text {min }}$. In order not to contradict our second assumption, the radiative deceleration timescale for an electron with the Lorentz factor $\gamma_{\text {min }}$ must be smaller than the GRB duration measured in the shock comoving frame. In this case, it can be shown that the contribution of electrons with $\gamma_{\mathrm{e}} \gg \gamma_{\min }$ or $\gamma_{\mathrm{e}} \ll \gamma_{\min }$ to GRB bolometric luminosity is small. It follows from the above consideration that the choice of electron injection spectrum does not matter unless the question about the exact shape of GRB spectrum is addressed; note that $\gamma_{\text {min }}$ in the approach of Sari et al. corresponds to $\gamma_{p}$ in ours and that the peak in the observed spectrum is at the energy determined by the electrons with $\gamma_{\mathrm{e}} \sim \gamma_{\mathrm{p}}$.

4. The energy dissipated in the shock is efficiently redistributed all over the shocked gas by large-scale turbulence and then transferred to radiating particles on a longer timescale. - We are considering a quasi-stationary problem, when the energy is stored in heavy particles 
(protons) and there is a continuous supply of relativistic electrons which replace those that have decelerated. The reason why we used a quasi-stationary formulation is that the bottle-neck in the energy transfer path (from protons to large-scale turbulence, then to electrons, then to radiation) is, most likely, at the first two chains, i.e., in a typical GRB, electrons are able to radiate at a much higher rate than they can gain energy from protons or large-scale turbulence.

5. The fireball is isotropic. - This is a good approximation even for a jet if it has an opening angle $\theta \gg \Gamma^{-1}$. If the central engine generates a jet-like outflow, then the value of $E_{\mathrm{GRB}}$ is not the actual GRB energy but rather the energy calculated assuming isotropic emission. Therefore, the unknown beaming factor does not affect our results.

\section{Physical parameters in GRB shocks}

For the synchrotron-self-Compton model one may represent the luminosity of a single highly relativistic electron in the following form (Ginzburg 1987; Rybicki \& Lightman 1979):

$\mathcal{L}\left(\gamma_{\mathrm{e}}\right)=\frac{4}{3} \gamma_{\mathrm{e}}^{2} c\left[\sigma_{\mathrm{T}}\left(w_{\mathrm{m}}+w_{\mathrm{lr}}\right)+\int \sigma w_{\mathrm{hr}, \omega} \mathrm{d} \omega\right]$.

Here $w_{\mathrm{m}}$ and $w_{\mathrm{lr}}$ are the energy densities of magnetic field and low-energy radiation, respectively, $w_{\mathrm{hr}, \omega}$ is the spectral energy density of high-energy radiation, $\sigma_{\mathrm{T}}$ the Thomson scattering cross-section. The boundary between low-energy and high-energy photons is the Klein-Nishina cut-off at $\hbar \omega \simeq m_{\mathrm{e}} c^{2} / \gamma_{\mathrm{e}}$. Above the cut-off, the transport cross-section $\sigma$ is a function of $\gamma_{\mathrm{e}} \omega$. It decreases as $\left(\gamma_{\mathrm{e}} \omega\right)^{-2}$ for $\gamma_{\mathrm{e}} \hbar \omega \gg m_{\mathrm{e}} c^{2}$ and approaches $\sigma_{\mathrm{T}}$ in the opposite limit.

The relative efficiency of Comptonization and synchrotron emission is determined by the Compton $y$ parameter (Sari et al. 1996)

$y=\sigma_{\mathrm{T}} L \int_{1}^{\infty}\left(\gamma_{\mathrm{e}}^{2}-1\right) n_{\mathrm{e}}\left(\gamma_{\mathrm{e}}\right) \mathrm{d} \gamma_{\mathrm{e}} \simeq \gamma_{\mathrm{p}}^{2} \sigma_{\mathrm{T}} n_{\mathrm{e}} L$,

where $n_{\mathrm{e}}$ is the number density of electrons with the Lorentz factors $\sim \gamma_{\mathrm{p}}$ and $L$ is the size of the emitting region. For example, when electromagnetic radiation passes through a layer filled with relativistic electrons and the energy of incident photons satisfies the relation $\varepsilon \ll m_{\mathrm{e}} c^{2} / \gamma_{\mathrm{e}}$ (the classical regime), the power of comptonized radiation relative to the power of incident radiation is given by $L_{\text {ic }} / L_{\text {in }} \equiv \tau_{\text {ic }} \simeq 4 y / 3$. Below, we will call $\tau_{\text {ic }}$ the optical depth for inverse Comptonization. In general, this relation contains a numerical factor which is different from $4 / 3$ and depends on the geometry of a source.

The condition $\tau_{\text {ic }}=1$ (together with $\gamma_{\mathrm{p}}<m_{\mathrm{e}} c^{2} / \varepsilon$ ) means that the source radiates via inverse Compton scattering as much energy as via the other emission mechanisms. If the comptonized photons may in turn scatter off electrons in classical regime (i.e., their energy is below the Klein-Nishina cut-off), the cascade Comptonization is possible. For any $\tau_{\text {ic }}<1$ most of the energy will be carried by incident radiation. Otherwise, the largest fraction of bolometric luminosity is due to $k$ times comptonized photons, where $k$ is the minimum number of consequent upscatterings, which rises an average photon energy above the Klein-Nishina cut-off. The latter statement is valid when the integral term in Eq. (1) is ignored. This is possible even for $w_{\mathrm{hr}} \gg w_{\mathrm{lr}}$ thanks to a very rapid decrease in the transport cross-section $\sigma$. It should be noted, however, that this simplification may not be applied in some cases, as discussed below.

Let us consider a relativistic shock with the Lorentz factor $\Gamma$ which radiates an energy $E_{\mathrm{r}}$ during a time interval $\tau$ (in the observer frame). The values of $E_{\mathrm{r}}$ and $\tau$ are either the total GRB energy $E_{\mathrm{GRB}}$ and duration $t_{\mathrm{GRB}}$ (in the external shock model), or the energy and duration of individual pulses (in the model of internal shocks), which constitute together what we call a GRB. As measured in the comoving frame, bolometric luminosity of the source is $E_{\mathrm{r}} / \Gamma^{2} \tau$. Given the expression for the integral luminosity of a single particle (1), one easily arrives at the following relation:

$\frac{E_{\mathrm{r}}}{\Gamma^{2} \tau}=\frac{4}{3} \gamma_{\mathrm{p}}^{2} \sigma_{\mathrm{T}} N_{\mathrm{e}} c\left(w_{\mathrm{m}}+w_{\mathrm{lr}}\right)$,

where $N_{\mathrm{e}}$ is the total number of electrons with $\gamma_{\mathrm{e}} \sim \gamma_{\mathrm{p}}$ in the GRB shell. The product $N_{\mathrm{e}}\left(w_{\mathrm{m}}+w_{\mathrm{lr}}\right)$ may be represented in the form $n_{\mathrm{e}}\left(W_{\mathrm{m}}+W_{\mathrm{lr}}\right)$, where $W_{\mathrm{m}}$ and $W_{\text {lr }}$ are the total energy of magnetic field and low-energy radiation in the emitting region. Certainly, the sum $W_{\mathrm{m}}+W_{\mathrm{lr}}$ cannot exceed a fraction of the bulk kinetic energy which is transferred to the particles at the shock front. According to the assumption 2, this fraction, $E_{i}$, is of the order of $E_{\mathrm{r}} / \Gamma$, where the factor $\Gamma$ appears because $E_{i}$ is measured in the comoving frame.

Most of the remaining factors in the right-hand side of Eq. (3) can be substituted from Eq. (2), where the thickness of the emitting region behind a relativistic shock grows during the time interval $\tau$ up to $L \sim \Gamma c \tau$ (in the comoving frame). Finally, Eq. (3) yields

$\tau_{\text {ic }} \gtrsim \frac{E_{\mathrm{r}} / \Gamma}{E_{i}} \sim 1$

Thus, there is only one possibility to satisfy all the adopted assumptions: the observed sub-MeV emission from GRBs should be well above the Klein-Nishina cut-off for electrons in the emitting region in order not to generate too much ultra-hard radiation via the inverse Compton process.

To specify this requirement quantitatively, it is necessary to know the low-energy portion of the GRB spectrum. If it is a power-law with the index $\alpha\left(\omega I_{\omega} \propto \omega^{\alpha}\right)$, then according to assumption 1 we have

$$
\left(\frac{\gamma_{\mathrm{p}} \varepsilon_{\mathrm{p}}}{\Gamma m_{\mathrm{e}} c^{2}}\right)^{\alpha^{\prime}} \gtrsim \tau_{\mathrm{ic}} \quad \Rightarrow \quad \gamma_{\mathrm{p}} \gtrsim 2.5 \Gamma \tau_{\mathrm{ic}}^{1 / \alpha^{\prime}}
$$

where we use the fact that the observed spectrum of an average GRB has a maximum at $\varepsilon_{\mathrm{p}} \sim 200 \mathrm{keV}$. In Eq. (5) $\alpha^{\prime}=\min \{\alpha, 2\}$ : for spectra rising steeper than $\omega^{2}$ the 
contribution of photons near $\varepsilon_{\mathrm{p}}$ to the inverse Compton cooling rate dominates, despite a much smaller transport cross-section $\sigma$.

When synchrotron-self-Compton mechanism is the main one operating in the source, the maximum of the observed GRB spectrum is at

$\varepsilon_{\mathrm{p}} \sim 0.4 \Gamma\left(\frac{4}{3} \gamma_{\mathrm{p}}^{2}\right)^{k+1} \frac{\hbar e B}{m_{\mathrm{e}} c}$

where $k$ is the number of Comptonization cascades; $k=0$ corresponds to the synchrotron radiation. The magnetic field strength may be estimated from Eq. (3), where one has to establish the relation between $w_{\mathrm{m}}$ and $w_{\mathrm{lr}}$. Given the expression (1) for the synchrotron luminosity $\left(w_{\mathrm{lr}}=w_{\mathrm{hr}}=0\right)$ of a single particle, the energy density of synchrotron radiation is

$w_{\mathrm{sy}} \simeq \frac{4}{3} \gamma_{\mathrm{p}}^{2} \sigma_{\mathrm{T}} c \frac{B^{2}}{8 \pi} n_{\mathrm{e}} \frac{L}{c}=\tau_{\mathrm{ic}} w_{\mathrm{m}}$.

This relation follows directly from Eq. (1). It is well-known that each subsequent cascade of Comptonization also leads to a $\tau_{\text {ic }}$ times increase in the energy density of comptonized photons. Finally, one obtains

$w_{\mathrm{lr}}+w_{\mathrm{m}}=\sum_{j=0}^{k} \tau_{\mathrm{ic}}^{j} \frac{B^{2}}{8 \pi} \simeq \tau_{\mathrm{ic}}^{k} \frac{B^{2}}{8 \pi}$,

where the approximate equality is for $\tau_{\text {ic }} \gg 1$.

After some algebra, i.e., deriving the magnetic field strength from Eq. (8) with the help of the relation (3) and substituting $B$ in Eq. (6), one has the following result

$\gamma_{\mathrm{p}} \simeq \frac{\sqrt{3}}{2} \tau_{\mathrm{ic}}^{\frac{1}{4}} \Gamma^{\frac{1}{k+1}}\left(\frac{2.5 m_{\mathrm{e}} c \varepsilon_{\mathrm{p}}}{e \hbar}\right)^{\frac{1}{2(k+1)}}\left(\frac{c^{3} \tau^{3}}{2 E_{\mathrm{r}}}\right)^{\frac{1}{4(k+1)}}$

which should be compared with the requirement (5). Thus,

$\tau_{\mathrm{ic}}^{\frac{1}{\alpha^{\prime}}-\frac{1}{4}} \lesssim \frac{\sqrt{3} \varepsilon_{\mathrm{p}}}{2 m_{\mathrm{e}} c^{2}} \Gamma^{-\frac{k}{k+1}}\left(\frac{2.5 m_{\mathrm{e}} c \varepsilon_{\mathrm{p}}}{e \hbar}\right)^{\frac{1}{2(k+1)}}\left(\frac{c^{3} \tau^{3}}{2 E_{\mathrm{r}}}\right)^{\frac{1}{4(k+1)}}$.

Because we know that $\alpha^{\prime} \leq 2$ and $\tau_{\text {ic }} \gtrsim 1$ (see Eq. (4)), the conclusion is straightforward: $k=0$, except the extreme case

$$
\begin{aligned}
\Gamma & \lesssim \frac{3 \varepsilon_{\mathrm{p}}^{2}}{4 m_{\mathrm{e}}^{2} c^{4}}\left(\frac{2.5 m_{\mathrm{e}} c \varepsilon_{\mathrm{p}}}{e \hbar}\right)^{1 / 2}\left(\frac{c^{3} \tau^{3}}{2 E_{\mathrm{r}}}\right)^{1 / 4} \\
& \sim 25 \frac{t_{1}^{3 / 4}}{E_{52}^{1 / 4}}\left(\frac{\tau}{t_{\mathrm{GRB}}}\right)^{1 / 2} .
\end{aligned}
$$

That is, the radiation which is observed from GRBs in the sub-MeV spectral range is generated by a synchrotron mechanism.

Substituting $k=0$ into Eq. (10) we find that the value of $\tau_{\text {ic }}$ must fit in the following window

$$
1 \lesssim \tau_{\mathrm{ic}}^{\frac{1}{\alpha^{\prime}}-\frac{1}{4}} \lesssim 300\left(\frac{\varepsilon_{\mathrm{p}}}{m_{\mathrm{e}} c^{2}}\right)^{\frac{3}{2}}\left(\frac{t_{1}^{3}}{E_{52}}\right)^{\frac{1}{4}}\left(\frac{\tau}{t_{\mathrm{GRB}}}\right)^{\frac{1}{2}}
$$

where $t_{1}$ is the duration of GRB in units of $10 \mathrm{~s}, E_{52}$ the burst energy in units of $10^{52} \mathrm{erg}$, and for simplicity the luminosity in peaks, $E_{\mathrm{r}} / \tau$, is assumed to be of the order of the average luminosity, $E_{\mathrm{GRB}} / t_{\mathrm{GRB}}$. Equation (12) gives a lot of freedom for $\alpha^{\prime} \simeq 2$. However, we suppose that the spectrum is much flatter, with $\alpha$ not larger than unity, which is true for the majority of GRBs (Tavani 1996). In this case, the result of our analysis becomes more definite: the optical depth for inverse Comptonization is between 1 and 30, approaching 100 in extreme cases. Consequently, the magnetic field strength $B$ is rather close to its equipartition value (here we mean the equipartition with the energy stored in protons),

$B \simeq\left[\frac{2 E_{\mathrm{r}}}{\left(\Gamma^{2} c \tau\right)^{3} \tau_{\mathrm{ic}}}\right]^{1 / 2} \sim \frac{E_{52}^{1 / 2}}{t_{1}^{3 / 2} \tau_{\mathrm{ic}}^{1 / 2}} \frac{t_{\mathrm{GRB}}}{\tau} \frac{10^{9}}{\Gamma^{3}} \mathrm{G}$.

An estimate of $\gamma_{p}$ may be obtained from Eq. (9):

$\gamma_{\mathrm{p}} \simeq 200 \tau_{\mathrm{ic}}^{1 / 4} \Gamma\left(\frac{t_{1}^{3}}{E_{52}}\right)^{\frac{1}{4}}\left(\frac{\tau}{t_{\mathrm{GRB}}}\right)^{\frac{1}{2}}$.

A significant fraction of the total GRB energy is converted via inverse Compton scattering into ultra-hard emission. The typical energy of comptonized photons corresponds to the Klein-Nishina cut-off at

$\varepsilon_{\mathrm{ic}} \sim \Gamma \gamma_{\mathrm{p}} m_{\mathrm{e}} c^{2} \sim 10^{-4} \Gamma^{2}\left(\frac{t_{1}^{3}}{E_{52}}\right)^{\frac{1}{4}}\left(\frac{\tau}{t_{\mathrm{GRB}}}\right)^{\frac{1}{2}} \mathrm{TeV}$

in the observer frame. The ratio of energy carried by the ultra-hard radiation to the energy carried by the sub-MeV radiation is

$$
\begin{aligned}
\delta E_{\mathrm{ic}} & \simeq\left(\frac{\Gamma m_{\mathrm{e}} c^{2}}{\gamma_{\mathrm{p}} \varepsilon_{\mathrm{p}}}\right)^{\alpha} \tau_{\mathrm{ic}} \\
& \gtrsim\left[1.3 \times 10^{-2}\left(\frac{E_{52}}{t_{1}^{3}}\right)^{1 / 4}\left(\frac{t_{\mathrm{GRB}}}{\tau}\right)^{1 / 2}\right]^{\alpha}
\end{aligned}
$$

For sufficiently flat spectra $(\alpha<1)$, which are considered here, the relative energy content of ultra-hard radiation is always non-negligible. In the external shock model one has $\delta E_{\mathrm{ic}} \gtrsim 1 \%$. Moreover, in the model of internal shocks, in which $\tau \sim 1-10 \mathrm{~ms}$ (presumably), the value of $\delta E_{\mathrm{ic}}$ should be close to its limiting value of unity set by the assumption 1 , independently on the spectral index $\alpha$.

\section{Implications of the analysis}

The principle outcome of the above analysis is that the observed sub-MeV emission in GRBs is generated by the synchrotron mechanism. However, the limits given in Eq. (12) become mutually exclusive if

$\left(\frac{\varepsilon_{\mathrm{p}}}{m_{\mathrm{e}} c^{2}}\right)^{3}\left(\frac{t_{1}^{3}}{E_{52}}\right)^{\frac{1}{2}} \frac{\tau}{t_{\mathrm{GRB}}}<10^{-5}$.

The bursts having parameters in this domain can radiate only a minor part of the available energy in the sub-MeV spectral range. Therefore, Eq. (17) defines the 
synchrotron-self-Compton (SSC) constraint for GammaRay Bursts.

There are two implications of the SSC constraint. It may be treated as a lower limit on GRB duration (the external shock model, $\tau=t_{\mathrm{GRB}}$ ):

$t_{\mathrm{GRB}}>5 \times 10^{-3} E_{52}^{1 / 3}\left(\frac{m_{\mathrm{e}} c^{2}}{\varepsilon_{\mathrm{p}}}\right)^{2} \mathrm{~s} \sim 0.03 \mathrm{~s}$.

On the other hand, Eq. (17) determines the shortest possible variability timescale $\tau$ in the model of internal shocks:

$\tau>10^{-5}\left(\frac{m_{\mathrm{e}} c^{2}}{\varepsilon_{\mathrm{p}}}\right)^{3}\left(\frac{E_{52}}{t_{1}^{3}}\right)^{\frac{1}{2}} t_{\mathrm{GRB}} \sim 10^{-3} \mathrm{~s}$.

A variability significantly shorter than the limiting duration $1 \mathrm{~ms}$ - if observed in GRB lightcurves - favours propagation effects or coherent emission mechanisms as a primary explanation for short-time pulsations in GRBs.

In order not to contradict assumption 2, the deceleration timescale for an electron with the Lorentz factor $\gamma_{\mathrm{p}}$ must be smaller than the GRB duration measured in the shock comoving frame. Since we know the relations between $B, \gamma_{\mathrm{p}}$ and the parameters characterizing a GRB ( $E_{\mathrm{GRB}}, t_{\mathrm{GRB}}, \tau$ and $\Gamma$ ), it is possible to use this constraint to set limit on $\Gamma$ :

$$
\begin{aligned}
\Gamma & \lesssim\left(\frac{\sigma_{\mathrm{T}}}{4 \pi \sqrt{3}}\right)^{\frac{1}{4}}\left(\frac{2.5 \varepsilon_{\mathrm{p}}}{e \hbar m_{\mathrm{e}} c}\right)^{\frac{1}{8}}\left(\frac{2 E_{\mathrm{GRB}}}{\tau_{\mathrm{ic}} c^{3} t_{\mathrm{GRB}}^{3}}\right)^{\frac{3}{16}}\left(\frac{t_{\mathrm{GRB}}}{\tau}\right)^{\frac{1}{8}} t_{\mathrm{GRB}}^{\frac{1}{4}} \\
& \simeq 1.2 \times 10^{3} \frac{E_{52}^{3 / 16}}{\tau_{\mathrm{ic}}^{3 / 16} t_{1}^{5 / 16}}\left(\frac{t_{\mathrm{GRB}}}{\tau}\right)^{\frac{1}{8}} .
\end{aligned}
$$

Exactly the same limit may be derived from the requirement that the total energy of the accelerated electrons does not exceed the GRB energy. It follows from the above analysis that the accelerated electrons, in the general case, are not in equipartition with the magnetic field.

An important question to be addressed in the theory of synchrotron emission is why the value of $\varepsilon_{\mathrm{p}}$ is nearly invariant. This problem may be reformulated as a question regarding the electron acceleration mechanism. The simplest reasonable suggestion is that $\gamma_{\mathrm{p}}$ is a function of magnetic field strength $B$, which is given by Eq. (13). To eliminate $\Gamma$-dependence (the strongest one in Eq. (6)), the acceleration mechanism should provide $\gamma_{\mathrm{p}} \propto B^{-1 / 3}$. In this case, $\varepsilon_{\mathrm{p}}$ preserves a weak dependence on $E_{\mathrm{r}}$ and $\tau, \varepsilon_{\mathrm{p}} \propto E_{\mathrm{r}}^{1 / 6} \tau^{-1 / 2}$, where the dependence on $\tau$ is likely to be hidden by a stronger effect of the cosmological time dilation.

Several per cent (a few tens per cent is a more likely figure) of the total GRB energy must be converted in the ultra-hard spectral domain via the inverse Compton scattering. The location of the maximum in the spectrum of comptonized radiation is model-dependent. In the model of internal shocks, the maximum (see Eq. (15)) may be at energies as low as $10^{-6} \Gamma^{2} \mathrm{TeV} \sim 10-100 \mathrm{GeV}$, so that the number fluence of ultra-hard photons should be of the order of $10^{3} \mathrm{~km}^{-2}$ even for the weakest bursts. However, the uncertainty in theoretical predictions is large: the maximum in the spectrum of inverse Compton radiation may be located at $\sim 10^{3} \mathrm{TeV}$. On the other hand, in the external shock model, the location of the maximum is more definite, namely, $\sim 10^{-4} \Gamma^{2} \mathrm{TeV} \sim 1-100 \mathrm{TeV}$. In this case the ultra-hard emission is inaccessible for direct observation because photons above several hundred $\mathrm{GeV}$ are strongly absorbed by the infrared background radiation (Primack et al. 1999), and the observed fluence below $1 \mathrm{TeV}$ is determined by the spectral slope of comptonized radiation in $\mathrm{TeV}$ energy range. To date, there is only one indication of sub-TeV emission accompanying GRBs (Atkins et al. 2000).

According to our estimates, in both models, the GRB source may be optically thick for the two-photon absorption of the highest energy quanta. Qualitative results of the two-photon absorption are the following (details will be discussed elsewhere). Each of the absorbed photons produces an electron-positron pair, in which the daughter particles have roughly equal energies. Electrons and positrons have a larger interaction cross-section than photons have. Therefore, if the optical depth for interaction of initial ultra-hard quanta is larger than unity, the same is true for daughter electrons (positrons). The first photon scattered off by one of these energetic particles takes away about a half of its energy, and in turn may be absorbed. Step by step, the radiation becomes softer until the absorption threshold is reached. It is this spectral domain where the bulk of energy initially contained in the ultrahard radiation is concentrated. If the absorption threshold is below $1 \mathrm{TeV}$, then the reprocessed radiation may reach the Earth. A similar picture arises if one considers the reprocessing of the ultra-hard photons via interaction with soft GRB radiation scattered in interstellar medium (Derishev et al. 2000).

The result $\tau_{\text {ic }} \sim 1-30$ seems surprising since $\tau_{\text {ic }}$ is an integral characteristic of the emitting region, while it depends on $\gamma_{p}$, which is determined by the balance between acceleration and losses and, therefore, is defined by local conditions. Such a fine tuning in the external shock model may result from the following. We have already noted that the statement $\tau_{\text {ic }}=1$ means that the energy densities of the magnetic field and synchrotron radiation are equal in the emitting region. Since this region expands with a relativistic velocity, a large fraction of newly born synchrotron photons cannot escape it until a GRB shell as a whole is decelerated to a Lorentz factor much smaller than the initial one. During the initial deceleration stage the synchrotron radiation is accumulated to the point at which its energy density becomes nearly equal to the thermal energy density of plasma. If strong turbulence in a GRB shell rapidly builds up a magnetic field of a strength close to the equipartition value, then the approximate correspondence between the energy density of magnetic field and that of synchrotron radiation is established automatically, thus explaining why $\tau_{\text {ic }} \sim 1$. In the model of internal shocks, it is usually assumed that the Lorentz factor of successive shells differs by a factor of few. Nearly all generated 
radiation remains within the emitting region and the condition $\tau_{\text {ic }} \sim 1$ is satisfied automatically.

The situation when $\tau_{\text {ic }}>1$ and, at the same time, the synchrotron emission is the most efficient cooling mechanism in the portion of electron distribution with the highest luminosity yields the largest flexibility in spectral shapes of synchrotron radiation. So far, we considered the low-energy spectral slope as an independent parameter, assuming only that $\alpha \leq 2$. The complete theory of synchrotron-self-Compton emission, however, should give a definite prediction of $\alpha$.

In the simplest approximation only the synchrotron losses are taken into account, so that partially decelerated electrons form the following distribution below $\gamma_{\mathrm{p}}$ :

$n_{\mathrm{e}}\left(\gamma_{\mathrm{e}}\right)=\frac{m_{\mathrm{e}} c^{2}}{\mathcal{L}\left(\gamma_{\mathrm{e}}\right)} \frac{\mathrm{d} n_{\mathrm{e}}}{\mathrm{d} t}=n_{\mathrm{e}} \frac{\gamma_{\mathrm{p}}}{\gamma_{\mathrm{e}}^{2}}$

For a power-law electron distribution, $n_{\mathrm{e}}\left(\gamma_{\mathrm{e}}\right) \propto \gamma_{\mathrm{e}}^{q}$, the synchrotron spectrum is also a power-law, $\omega I_{\omega} \propto \omega^{\frac{q+3}{2}}$. Distribution (21) gives the well-known result, $\omega I_{\omega} \propto \omega^{1 / 2}$.

A different approximation works well when the integral term in Eq. (1) dominates. In this case, the main contribution to the integral is from photons near the Klein-Nishina cut-off whose frequency scales as $\gamma_{\mathrm{e}}^{-1}$. Looking for a powerlaw solution, we find that $\mathcal{L} \propto \gamma_{\mathrm{e}}^{2-\alpha}$ if $\omega I_{\omega} \propto \omega^{\alpha}$, and that $n_{\mathrm{e}}\left(\gamma_{\mathrm{e}}\right) \propto \gamma_{\mathrm{e}}^{\alpha-2}$ consequently. Thus, $\alpha=(\alpha+1) / 2$ and the final result is

$n_{\mathrm{e}}\left(\gamma_{\mathrm{e}}\right)=\frac{n_{\mathrm{e}}}{\gamma_{\mathrm{e}}} \quad$ and $\quad I_{\omega}=$ const.

As follows from the above analysis, the GRB sources are at the border between these two limiting cases. Namely, the synchrotron losses dominate for electrons with $\gamma_{\mathrm{e}} \sim \gamma_{\mathrm{p}}$, but the inverse Compton losses become dominant for less energetic electrons because the Klein-Nishina cut-off is shifted to higher photon energies, giving greater $w_{\mathrm{lr}}$. The fact that all GRBs belong to the transition zone between two limiting cases makes theoretical analysis more complicated, but also allows for a wider range of solutions.

Let us consider one example to illustrate this. Suppose that electrons with the Lorentz factors between $\gamma_{\mathrm{ic}}$ and $\gamma_{\mathrm{p}}$ lose their energy mainly due to synchrotron radiation. The electrons in this range form the distribution (21) and produce the corresponding spectrum $\omega I_{\omega} \propto \omega^{1 / 2}$, which extends from $\varepsilon_{\mathrm{p}}$ down to $\left(\gamma_{\mathrm{ic}} / \gamma_{\mathrm{p}}\right)^{2} \varepsilon_{\mathrm{p}}$. If the Klein-Nishina cut-off, $\varepsilon_{\mathrm{k}-\mathrm{n}}$, lies somewhere between $\left(\gamma_{\mathrm{ic}} / \gamma_{\mathrm{p}}\right)^{2} \varepsilon_{\mathrm{p}}$ and $\left(\gamma_{\mathrm{ic}} / \gamma_{\mathrm{p}}\right) \varepsilon_{\mathrm{p}}$ for $\gamma_{\mathrm{e}} \sim \gamma_{\mathrm{p}}$, then the relative importance of the integral term in Eq. (1) grows until $\gamma_{\mathrm{e}}<\gamma_{\mathrm{k}-\mathrm{n}}=\varepsilon_{\mathrm{k}-\mathrm{n}} / \varepsilon_{\mathrm{p}}$. Electron distribution between $\gamma_{\mathrm{k}-\mathrm{n}}$ and $\gamma_{\mathrm{ic}}$ is determined by the spectral slope in the region just below $\varepsilon_{\mathrm{p}}$, where, in this case, the spectral index is $\alpha=1 / 2$. Hence, electron distribution is given by $n_{\mathrm{e}}\left(\gamma_{\mathrm{e}}\right) \propto \gamma_{\mathrm{e}}^{-3 / 2}$ (see the discussion of Eq. (22)) and the synchrotron spectrum generated by these electrons has an index $\alpha=3 / 4$ between energies $\left(\gamma_{\mathrm{ic}} / \gamma_{\mathrm{p}}\right)^{2} \varepsilon_{\mathrm{p}}$ and $\left(\gamma_{\mathrm{k}-\mathrm{n}} / \gamma_{\mathrm{p}}\right)^{2} \varepsilon_{\mathrm{p}}$. At the lowest energies, the spectral index is again $\alpha=1 / 2$, since the inverse Compton losses saturate when the Klein-Nishina cut-off is shifted above $\varepsilon_{\mathrm{p}}$.
The GRB spectrum below $\varepsilon_{\mathrm{p}}$ is a broken power-law consisting of two portions with the spectral index $1 / 2$ separated by a steeper part with $\alpha=3 / 4$. Therefore, in addition to the well-known cooling break in the synchrotron spectrum there are two others - the lower-energy KleinNishina break, below which the ratio of synchrotron to inverse Compton losses levels off, and the higher-energy Compton break, above which synchrotron losses overcome inverse Compton ones. Locations of all spectral breaks are time-dependent both during the main pulse and at the afterglow stage, and if one is limited to a narrow-band observations, the transition from one spectral slope to another could be interpreted as a change in the decline rate of a lightcurve. A similar effect may appear for a bolometric lightcurve when the balance between synchrotron and inverse Compton emissivities is shifted in favor of the latter.

One more effect may alter the appearance of the lowenergy part of the GRB spectrum - the synchrotron selfabsorption. With the help of expressions for $\gamma_{\mathrm{p}}$ and $\tau_{\text {ic }}$, it is not difficult to estimate the self-absorption threshold energy (Zheleznyakov 1996). It takes the highest value in the model of internal shocks, but even in this model the threshold is hardly above $500 \mathrm{eV}$ in the observer frame.

\section{Conclusions}

The analysis in this paper relies to a large extent upon several assumptions which have not received yet direct observational confirmation. However, we put forward a number of reasons why these assumptions are consistent with the known properties of GRBs. Our investigation reveals that, in the range of parameters typical for the detected bursts, there is no other possibility that accounts for the sub-MeV radiation than synchrotron emission of electrons accelerated in relativistic shocks. On this basis, we make several definite predictions of physical parameters in relativistic shocks of GRB origin.

One of the most important conclusions is that physical conditions in GRB emitting regions are qualitatively similar. Namely, there is not so much freedom in the magnetic field strength, which is close to its equipartition value, and in the Lorentz factor of accelerated electrons (Eq. (9)). The main uncertainty in the energy of comptonized photons arises from differences between external and internal shock models and from poor knowledge of the bulk Lorentz factor. The inverse Compton losses always play a significant role, so that the ultra-hard emission above $100 \mathrm{GeV}$ contains from several to tens per cent of the total GRB energy.

We demonstrate that a steady-state electron distribution consistent with the Compton losses may produce different spectral indices, ranging from the widely accepted $1 / 2$ to 1 . In many GRBs, the competition between inverse Compton and synchrotron losses should produce rather complex (power-laws with several breaks) spectra in the X-ray and soft $\gamma$-ray ranges. The time evolution of spectral breaks, as well as transition from predominantly 
synchrotron losses to predominantly inverse Compton losses, may account for the changes in the decline rate observed in the lightcurves of several GRB afterglows.

The prevalence of a synchrotron emission mechanism in GRBs imposes the strict limitation on the burst parameters, which may be called a synchrotron-self-Compton constraint. The physical meaning of this constraint is that there cannot be radiatively efficient bursts with arbitrary small duration or arbitrary fast variability. The requirement of high radiative efficiency also limits the Lorentz factor of GRB fireballs to a value not larger than $\sim 10^{3}-10^{4}$, depending on the model (external or internal shocks, respectively). So, the range of admissible Lorentz factors in the GRB case is limited from both sides.

Acknowledgements. This work has been supported by Russian Foundation for Basic Research (the project 99-02-18244) and by the Russian Academy of Science through a grant for young scientists.

\section{References}

Akerlof, C., et al. 1999, Nature, 398, 400

Atkins, R., et al. 2000, ApJ, 533, L119

Baring, M. G., \& Harding, A. K. 1995, Adv. Space Res., 15(5), 153

Covino, S., et al. 1999, A\&A, 348, L1

Derishev, E. V., Kocharovsky, V. V., \& Kocharovsky, Vl. V. 1999, ApJ, 521, 640
Derishev, E. V., Kocharovsky, V. V., \& Kocharovsky, Vl. V. 2000, Proceedings of the 5th Huntsville Gamma-Ray Burst Symposium, 18-22 October 1999, Huntsville, USA, p. 460

Frail, D. A., et al. 1997, Nature, 389, 261

Galama, T. J., et al. 1998, ApJ, 497, L13

Ginzburg, V. L. 1987, Theoretical physics and astrophysics (Nauka, Moscow)

Mészáros, P., Laguna, P., \& Rees, M. J. 1993, ApJ, 415, 181

Mészáros, P., Rees, M. J., \& Wijers, R. A. M. J. 1999, New Astr., 4, 303

Metzger, M. R., et al. 1997, Nature, 387, 878

Paczyński, B., \& Rhoads, J. E. 1993, ApJ, 418, L5

Panaitescu, A., Spada, M., \& Mészáros, P. 1999, ApJ, 522, L105

Piran, T. 1999, Phys. Rep., 314, 575

Primack, J. R., Bullock, J. S., Somerville, R. S., \& MacMinn, D. 1999, Astropart. Phys., 11, 93

Rees, M. J., \& Mészáros, P. 1992, MNRAS, 258, P41

Rees, M. J., \& Mészáros, P. 1994, ApJ, 430, L93

Reichart, D. E. 1997, ApJ, 485, L57

Reichart, D. E., \& Mészáros, P. 1997, ApJ, 483, 597

Rybicki, G. B., \& Lightman, A. P. 1979, Radiative Processes in Astrophysics

Sari, R., Narayan, R., \& Piran, T. 1996, ApJ, 473, 204

Sari, R., Piran, T., \& Narayan, R. 1998, ApJ, 497, L17

Shaviv, N., \& Dar, A. 1995, MNRAS, 277, 287

Tavani, M. 1996, ApJ, 466, 768

Waxman, E. 1997, ApJ, 485, L5

Wijers, R. A. M. J., et al. 1999, ApJ, 523, L33

Zheleznyakov, V. V. 1996, Radiation in astrophysical plasmas (Kluwer, Dordrecht/Boston/London) 